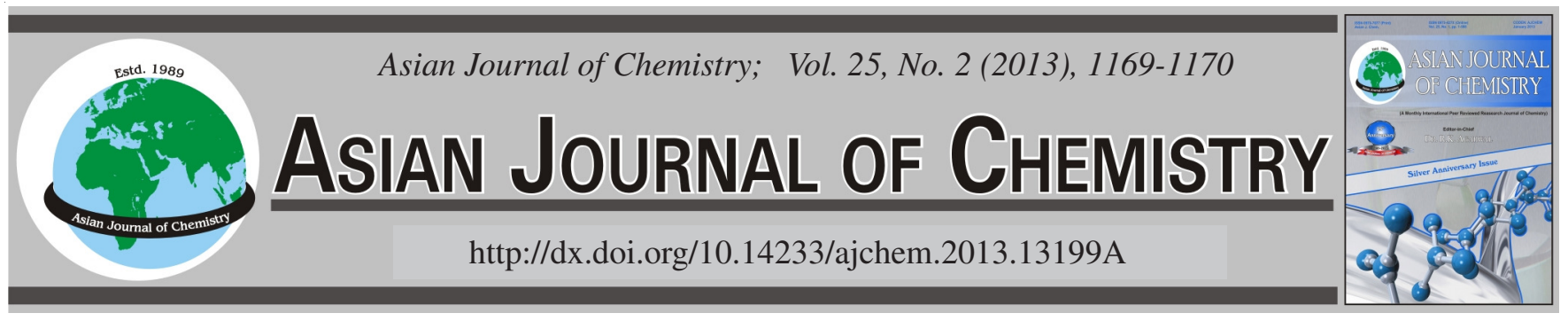

NOTE

\title{
Structural Aspects of Schiff Base Metal Complexes of Co(II), Ni(II) and Cu(II) Complexes Derived from 4-Hydroxy-1-ethyl quinoline
}

\author{
B.K. RaI ${ }^{1, *}$ and Arun KumaR ${ }^{2}$
}

${ }^{1}$ Department of Chemistry, L.N.T. College, Muzaffarpur-842 002, India

${ }^{2}$ Department of Chemistry, K.C.T.C. College, Raxaul-845 401, India

*Corresponding author: E-mail: binodkr_rai@yahoo.co.in

(Received: 7 December 2011;

Accepted: 31 August 2012)

AJC-12071

\begin{abstract}
Complexes of $\mathrm{Co}(\mathrm{II}), \mathrm{Ni}(\mathrm{II})$ and $\mathrm{Cu}(\mathrm{II})$ with 4-hydroxy-1-ethyl quinolin-2-(1H)-one hydrazone [HEQH] have been synthesized and characterized on the basis of their molar mass, elemental analyses, IR, UV, molar conductance and magnetic susceptibility measurements. On the basis of above physico-chemical and spectroscopic measurements it is proposed that the compound 4-hydroxy-1-ethyl quinolin$2-(1 H)$-one hydrazone acts in a bidentate ligand and co-ordination proposes through azomethine $\mathrm{N}$ atom and oxygen atom. The remaining valency of metal ions are satisfied by neutral molecules such as ammonia, pyridine, $\alpha, \beta$ or $\gamma$-picolines. Electronic spectral and magnetic susceptibility of the complexes proposes octahedral geometry for $\mathrm{Co}(\mathrm{II})$ and $\mathrm{Ni}(\mathrm{II})$ complexes. The geometry of the $\mathrm{Cu}$ (II) complexes is distorted octahedral in geometry.
\end{abstract}

Key Words: 4-Hydroxy-1-ethyl quinolin-2-(1H)-one hydrazone, $\mathrm{Co}(\mathrm{II}), \mathrm{Ni}(\mathrm{II}), \mathrm{Cu}(\mathrm{II}), \mathrm{Complexes.}$

Schiff bases are most widely used as chelating ligands in coordination chemistry ${ }^{1,2}$. They are also useful in catalysis, in medicine as antibiotics and to treat industrial waste. Semicarbazone Schiff bases are versatile ligands having biological importance as, antitumor agents, plant growth regulators, antibacterial, antineoplastic, antiviral, antileukemic and enzymatic reaction inhibitors. The complexes with such Schiff bases are very important due to their application in medicine, particularly in the chemotherapy of cancer ${ }^{3-5}$. In the view of above importance, we thought of interest to design Schiff bases having, nitrogen and oxygen architecture to bind the metal ions. in this communication, we report the synthesis, spectroscopic characterization of $\mathrm{Co}(\mathrm{II}), \mathrm{Ni}$ (II), $\mathrm{Cu}$ (II) complexes with Schiff base, 4-hydroxy-1-ethyl quinolin-2(1H)-one hydrazone [HEQH].

All the chemicals were from BDH or SRL. The metal, carbon, hydrogen nitrogen were estimated by standard methods. The magnetic susceptibility measurement were made at room temperature by Gouy method by using $\mathrm{Hg}\left[\mathrm{Co}(\mathrm{NCS})_{4}\right]$ as a calibrant. Melting point of the complexes were determined by open capillary method, IR spectra of the ligand as well as the metal complexes were recorded on Perkin-Elmer 577 spectrophotometer. The electronic spectra were measured on cary2390 spectrophotometer. Molar conductance were measured by systronics conductivity meter module $3 \mathrm{~B}$ in $\mathrm{DMF}$.
Preparation of the ligand: The ligand was synthesized refluxing the mixture of 4-hydroxy-1-ethyl quinolin-2(1H)one $(0.001 \mathrm{~m})$ dissolved in acetone and ethanolic solution of hydrazine hydrate $(0.01 \mathrm{~m})$. The light yellowish crystalline solid formed during refluxion was cooled, filtered, washed with ethanol and recrystallized from hot ethanol (yield $75 \%$ ).

Synthesis of $\mathrm{Co}(\mathrm{II}), \mathrm{Ni}$ (II) and $\mathrm{Cu}$ (II) complexes: The metal complexes were synthesized by adding ethanolic solution $(20 \mathrm{~mL})$ respective metals acetates of $\mathrm{Co}(\mathrm{II}), \mathrm{Ni}(\mathrm{II})$ and $\mathrm{Cu}(\mathrm{II})$ in presence of bases ammonia, puridine, $\alpha$-, $\beta$ - or $\gamma$ - picolines $(0.01 \mathrm{~m})$ to Schiff bases with each of metal separately. [HEQH] $(2.15 \mathrm{~g} 0.01 \mathrm{~m})$ in ethanol $(30 \mathrm{~mL})$. The reaction mixture was refluxed on a water bath for 3-4 h, on water bath. The partial removal of the solvent and cooling to room temperature gave solid coloured complexes which were filtered, washed thoroughly with ethanol and finally dried. Yield 60$65 \%$.

The vibrational spectra of the ligand as well as complexes has been recorded in Table-1 in the frequency range 4000$200 \mathrm{~cm}^{-1}$. The elemental analysis shows that all the complexes have 1:2 stoichiometry. The molar conductance values are too low to account for any dissociation of the complexes in DMF, indicating the non-electrolyte complexes (Table-2). The IR brands give valuable information regarding bonding modes of ligand to metal ions in the complexes. The IR spectrum of 


\begin{tabular}{|c|c|c|c|c|c|c|c|c|c|c|}
\hline \multirow{3}{*}{ Compound } & \multicolumn{9}{|c|}{$\begin{array}{l}\text { TABLE-2 } \\
\text { PHYSICOCHEMICAL MEASUREMENT OF LIGAND 4-HYDROXY-1-ETHYL QUINOLIN- } \\
\text { 2-(1H)-ONE HYDRAZONE (HEQH) AND ITS Co(II), Ni(II) AND Cu(II) COMPLEXES }\end{array}$} & \multirow{3}{*}{$\begin{array}{l}\Omega_{\mathrm{m}}\left(\mathrm{ohm}^{-1}\right. \\
\left.\mathrm{cm}^{2} \mathrm{~mol}^{-1}\right)\end{array}$} \\
\hline & \multirow{2}{*}{$\begin{array}{c}\text { Molar } \\
\text { mass }\end{array}$} & \multirow{2}{*}{$\begin{array}{l}\text { Yield } \\
(\%)\end{array}$} & \multicolumn{4}{|c|}{ Elemental analysis (\%): Found (calcd.) } & \multirow{2}{*}{$\begin{array}{c}\lambda_{\max } \text { electronic } \\
\left(\mathrm{cm}^{-1}\right)\end{array}$} & \multirow{2}{*}{$\begin{array}{l}\text { DT } \\
\left({ }^{\circ} \mathrm{C}\right)\end{array}$} & \multirow{2}{*}{$\mu_{\text {eff }}(\mathrm{BM})$} & \\
\hline & & & M & $\mathrm{C}$ & $\mathrm{H}$ & $\mathrm{N}$ & & & & \\
\hline HEQH & 215 & 75 & & $\begin{array}{c}66.80 \\
(66.97)\end{array}$ & $\begin{array}{c}5.92 \\
(8.84)\end{array}$ & $\begin{array}{c}19.45 \\
(19.53)\end{array}$ & - & - & - & - \\
\hline$\left[\mathrm{Co}(\mathrm{HEQH})_{2}\left(\mathrm{NH}_{3}\right)_{2}\right]$ & 554.93 & 63 & $\begin{array}{l}10.50 \\
10.61\end{array}$ & $\begin{array}{l}51.72 \\
51.89\end{array}$ & $\begin{array}{c}4.59 \\
(4.68)\end{array}$ & $\begin{array}{c}20.03 \\
(20.18)\end{array}$ & $\begin{array}{l}13300 \\
19400\end{array}$ & 223 & 4.96 & 3.4 \\
\hline$\left[\mathrm{Co}(\mathrm{HEQH})_{2}\left(\mathrm{C}_{6} \mathrm{H}_{5} \mathrm{~N}\right)_{2}\right]$ & 664.93 & 65 & $\begin{array}{l}8.48 \\
8.86\end{array}$ & $\begin{array}{l}43.20 \\
43.31\end{array}$ & $\begin{array}{c}3.87 \\
(3.91)\end{array}$ & $\begin{array}{c}16.73 \\
(16.84)\end{array}$ & $\begin{array}{l}13360 \\
18600\end{array}$ & 217 & 4.90 & 3.1 \\
\hline$\left[\mathrm{Co}(\mathrm{HEQH})_{2}(\alpha-\mathrm{pic})_{2}\right]$ & 672.93 & 65 & $\begin{array}{l}8.68 \\
8.75\end{array}$ & $\begin{array}{l}42.64 \\
42.79\end{array}$ & $\begin{array}{c}2.89 \\
(2.97)\end{array}$ & $\begin{array}{c}16.52 \\
(16.64)\end{array}$ & $\begin{array}{l}13500 \\
19100\end{array}$ & 242 & 4.92 & 2.8 \\
\hline$\left[\mathrm{Co}(\mathrm{HEQH})_{2}(\beta-\text { pic })_{2}\right]$ & 672.93 & 65 & $\begin{array}{l}8.69 \\
8.75\end{array}$ & $\begin{array}{l}42.65 \\
42.79\end{array}$ & $\begin{array}{c}2.92 \\
(2.97)\end{array}$ & $\begin{array}{c}16.53 \\
(16.64)\end{array}$ & $\begin{array}{l}13700 \\
18800\end{array}$ & 254 & 4.96 & 2.6 \\
\hline$\left[\mathrm{Co}(\mathrm{HEQH})_{2}(\gamma-\text { pic })_{2}\right]$ & 672.93 & 65 & $\begin{array}{l}8.67 \\
8.75\end{array}$ & $\begin{array}{l}42.67 \\
42.79\end{array}$ & $\begin{array}{c}2.93 \\
(2.97)\end{array}$ & $\begin{array}{l}16.57 \\
(16.64)\end{array}$ & $\begin{array}{l}13900 \\
18700\end{array}$ & 220 & 4.94 & 2.1 \\
\hline$\left[\mathrm{Ni}(\mathrm{HEQH})_{2}\left(\mathrm{NH}_{3}\right)_{2}\right]$ & 554.71 & 64 & $\begin{array}{l}10.49 \\
10.58\end{array}$ & $\begin{array}{l}51.80 \\
51.91\end{array}$ & $\begin{array}{c}4.62 \\
(4.68)\end{array}$ & $\begin{array}{c}20.06 \\
(.20 .19)\end{array}$ & $\begin{array}{c}11300,16000 \\
23900\end{array}$ & 210 & 3.18 & 1.8 \\
\hline$\left[\mathrm{Ni}(\mathrm{HEQH})_{2}\left(\mathrm{C}_{6} \mathrm{H}_{5} \mathrm{~N}\right)_{2}\right]$ & 664.71 & 65 & $\begin{array}{l}8.75 \\
8.83\end{array}$ & $\begin{array}{l}43.21 \\
43.32\end{array}$ & $\begin{array}{c}3.87 \\
(3.91)\end{array}$ & $\begin{array}{c}16.76 \\
(16.84)\end{array}$ & $\begin{array}{c}11260,15900 \\
23960\end{array}$ & 218 & 3.20 & 1.9 \\
\hline$\left[\mathrm{Ni}(\mathrm{HEQH})_{2}(\alpha-\mathrm{pic})_{2}\right]$ & 672.71 & 62 & $\begin{array}{l}8.64 \\
8.72\end{array}$ & $\begin{array}{l}42.70 \\
42.81\end{array}$ & $\begin{array}{c}3.89 \\
(3.95)\end{array}$ & $\begin{array}{c}16.58 \\
(16.64)\end{array}$ & $\begin{array}{c}11200,15800 \\
23990\end{array}$ & 202 & 3.22 & 2.2 \\
\hline$\left[\mathrm{Ni}(\mathrm{HEQH})_{2}(\beta-\text { pic })_{2}\right]$ & 672.71 & 60 & $\begin{array}{l}8.66 \\
8.72\end{array}$ & $\begin{array}{l}42.69 \\
42.81\end{array}$ & $\begin{array}{c}3.90 \\
(3.95)\end{array}$ & $\begin{array}{c}16.56 \\
(16.64)\end{array}$ & $\begin{array}{c}11100,16100 \\
24020\end{array}$ & 213 & 3.17 & 2.4 \\
\hline$\left[\mathrm{Ni}(\mathrm{HEQH})_{2}(\gamma-\mathrm{pic})_{2}\right]$ & 672.71 & 60 & $\begin{array}{l}8.67 \\
8.72\end{array}$ & $\begin{array}{l}42.67 \\
42.81\end{array}$ & $\begin{array}{c}3.91 \\
(3.95)\end{array}$ & $\begin{array}{c}16.50 \\
(16.64)\end{array}$ & $\begin{array}{c}11220,15980 \\
24000\end{array}$ & 217 & 3.21 & 2.5 \\
\hline$\left[\mathrm{Cu}(\mathrm{HEQH})_{2}\left(\mathrm{NH}_{3}\right)_{2}\right]$ & 555.54 & 61 & $\begin{array}{l}11.27 \\
11.35\end{array}$ & $\begin{array}{l}51.35 \\
51.17\end{array}$ & $\begin{array}{c}4.58 \\
(4.64)\end{array}$ & $\begin{array}{c}20.07 \\
(20.16)\end{array}$ & $13620-16770$ & 202 & 1.90 & 1.8 \\
\hline$\left[\mathrm{Cu}(\mathrm{HEQH})_{2}\left(\mathrm{C}_{6} \mathrm{H}_{5} \mathrm{~N}\right)_{2}\right]$ & 669.54 & 61 & $\begin{array}{l}9.40 \\
9.49\end{array}$ & $\begin{array}{l}42.80 \\
43.01\end{array}$ & $\begin{array}{c}3.81 \\
(3.88)\end{array}$ & $\begin{array}{l}16.61 \\
(1.72)\end{array}$ & $13620-16770$ & 201 & 1.92 & 1.7 \\
\hline$\left[\mathrm{Cu}(\mathrm{HEQH})_{2}(\alpha-\mathrm{pic})_{2}\right]$ & 677.54 & 61 & $\begin{array}{l}9.31 \\
9.37\end{array}$ & $\begin{array}{l}42.33 \\
42.50\end{array}$ & $\begin{array}{c}3.78 \\
(3.83)\end{array}$ & $\begin{array}{c}16.47 \\
(16.53)\end{array}$ & $13620-16770$ & 213 & 1.89 & 1.6 \\
\hline$\left[\mathrm{Cu}(\mathrm{HEQH})_{2}(\beta-\text { pic })_{2}\right]$ & 677.54 & 65 & $\begin{array}{l}9.30 \\
9.37\end{array}$ & $\begin{array}{l}42.35 \\
42.50\end{array}$ & $\begin{array}{c}3.79 \\
(3.83)\end{array}$ & $\begin{array}{c}16.44 \\
(16.53)\end{array}$ & $13620-16770$ & 212 & 1.93 & 1.4 \\
\hline$\left[\mathrm{Cu}(\mathrm{HEQH})_{2}(\beta-\text { pic })_{2}\right]$ & 677.54 & 65 & $\begin{array}{l}9.28 \\
9.37\end{array}$ & $\begin{array}{l}42.37 \\
42.50\end{array}$ & $\begin{array}{c}3.77 \\
(3.83)\end{array}$ & $\begin{array}{c}16.40 \\
(16.53)\end{array}$ & $13620-16770$ & 211 & 1.91 & 1.3 \\
\hline
\end{tabular}

TABLE-1

IR SPECTRAL BANDS OF LIGAND 4-HYDROXY-1-ETHYL QUINOLIN-2-(1H)-ONE HYDRAZONE (HEQH) AND ITS METAL COMPLEXES

\begin{tabular}{lcccc}
\hline \multicolumn{1}{c}{ Compounds } & $v(\mathrm{O}-\mathrm{H})$ & $v(\mathrm{C}=\mathrm{N})$ & $v(\mathrm{M}-\mathrm{O})$ & $v(\mathrm{M}-\mathrm{N})$ \\
\hline $\mathrm{HEQH}$ & $3260 \mathrm{~s}, \mathrm{~b}$ & $1560 \mathrm{~s}$ & - & - \\
{$\left[\mathrm{Co}(\mathrm{HEQH})_{2}\left(\mathrm{NH}_{3}\right)_{2}\right]$} & $3230 \mathrm{~m}, \mathrm{~b}$ & $1535 \mathrm{mb}$ & $515 \mathrm{~m}$ & $460 \mathrm{~m}$ \\
{$\left[\mathrm{Co}(\mathrm{HEQH})_{2}\left(\mathrm{C}_{6} \mathrm{H}_{5} \mathrm{~N}\right)_{2}\right]$} & $3230 \mathrm{~m}, \mathrm{~b}$ & $1535 \mathrm{~m}, \mathrm{~b}$ & $510 \mathrm{~m}$ & $465 \mathrm{~m}$ \\
{$\left[\mathrm{Co}(\mathrm{HEQH})_{2}(\alpha-\text { pic })_{2}\right]$} & $3235 \mathrm{~m}, \mathrm{~b}$ & $1530 \mathrm{~m}, \mathrm{~b}$ & $525 \mathrm{~m}$ & $465 \mathrm{~m}$ \\
{$\left[\mathrm{Co}(\mathrm{HEQH})_{2}(\beta-\text { pic })_{2}\right]$} & $3235 \mathrm{~m}, \mathrm{~b}$ & $1525 \mathrm{~m}, \mathrm{~b}$ & $530 \mathrm{~m}$ & $465 \mathrm{~m}$ \\
{$\left[\mathrm{Co}(\mathrm{HEQH})_{2}(\gamma-\mathrm{pic})_{2}\right]$} & $3235 \mathrm{~m}, \mathrm{~b}$ & $1535 \mathrm{~m}, \mathrm{~b}$ & $535 \mathrm{~m}$ & $465 \mathrm{~m}$ \\
{$\left[\mathrm{Ni}(\mathrm{HEQH})_{2}\left(\mathrm{NH}_{3}\right)_{2}\right]$} & $3230 \mathrm{~m}, \mathrm{~b}$ & $1530 \mathrm{~m},, \mathrm{~b}$ & $495 \mathrm{~m}$ & $470 \mathrm{~m}$ \\
{$\left[\mathrm{Ni}(\mathrm{HEQH})_{2}\left(\mathrm{C}_{6} \mathrm{H}_{5} \mathrm{~N}\right)_{2}\right]$} & $3225 \mathrm{~m}, \mathrm{~b}$ & $1535 \mathrm{mb}$ & $495 \mathrm{~m}$ & $460 \mathrm{~m}$ \\
{$\left[\mathrm{Ni}(\mathrm{HEQH})_{2}(\alpha-\text { pic })_{2}\right]$} & $3230 \mathrm{~m}, \mathrm{~b}$ & $1530 \mathrm{~m}, \mathrm{~b}$ & $495 \mathrm{~m}$ & $465 \mathrm{~m}$ \\
{$\left[\mathrm{Ni}(\mathrm{HEQH})_{2}(\beta-\mathrm{pic})_{2}\right]$} & $3230 \mathrm{~m}, \mathrm{~b}$ & $1525 \mathrm{~m}, \mathrm{~b}$ & $495 \mathrm{~m}$ & $470 \mathrm{~m}$ \\
{$\left[\mathrm{Ni}(\mathrm{HEQH})_{2}(\gamma-\mathrm{pic})_{2}\right]$} & $3230 \mathrm{~m}, \mathrm{~b}$ & $1535 \mathrm{mb}$ & $495 \mathrm{~m}$ & $470 \mathrm{~m}$ \\
{$\left[\mathrm{Cu}(\mathrm{HEQH})_{2}(\mathrm{NH})_{2}\right]$} & $3230 \mathrm{mb}$ & $1530 \mathrm{~m}, \mathrm{~b}$ & $510 \mathrm{~m}$ & $475 \mathrm{~m}$ \\
{$\left[\mathrm{Cu}(\mathrm{HEQH})_{2}\left(\mathrm{C}_{6} \mathrm{H}_{5} \mathrm{~N}\right)_{2}\right]$} & $3225 \mathrm{mb}$ & $1525 \mathrm{mb}$ & $505 \mathrm{~m}$ & $475 \mathrm{~m}$ \\
{$\left[\mathrm{Cu}(\mathrm{HEQH})_{2}(\alpha-\mathrm{pic})_{2}\right]$} & $3230 \mathrm{~m}$ & $1530 \mathrm{mb}$ & $510 \mathrm{~m}$ & $475 \mathrm{~m}$ \\
{$\left[\mathrm{Cu}(\mathrm{HEQH})_{2}(\beta-\mathrm{pic})_{2}\right]$} & $3225 \mathrm{~m}$ & $1535 \mathrm{mb}$ & $510 \mathrm{~m}$ & $475 \mathrm{~m}$ \\
{$\left[\mathrm{Cu}(\mathrm{HEQH})_{2}(\gamma-\mathrm{pic})_{2}\right]$} & $3225 \mathrm{~m}$ & $1530 \mathrm{~m}, \mathrm{~b}$ & $515 \mathrm{~m}$ & $475 \mathrm{~m}$ \\
\hline
\end{tabular}

the free ligand was compared with the spectra of the metal complexes. The IR spectrum of the Schiff bases strong ${ }^{6}$ and broad band at $3260 \mathrm{~cm}^{-1}$ assigned to no-N stretching. The shifting of this band to ${ }^{-1}$ lower wave number by $20-30 \mathrm{~cm}^{-1}$ indicates involvement of 0 atom of hydroxyl group in banding. The strong band observed at $1560 \mathrm{~cm}^{-1}$, indicating participation of azomethine nitrogen in coordination. The intense band at 1100 $1020 \mathrm{~cm}^{-1}$ in the complexes have been taken to be characteristic vibration of coordination pyridines and picolines molecules. The proposal coordination through oxygen atom of hydroxyl group is further supported by the appearance of a far ir band at $535-495 \mathrm{~cm}^{-1}$ assigned ${ }^{7}$ to $v(\mathrm{M}-\mathrm{O})$. The linkage with azomethine nitrogen is further confirmed by the appearance of another band in far $^{14}$ IR region at $470-450 \mathrm{~cm}^{-1}$ assigned to $v(M-N)$. The medium intensity band at $690-650 \mathrm{~cm}^{-1}$ in the complexes suggesting assign due to pyridine and picolines molecules.

\section{REFERENCES}

1. G. Wilkinson, Comprehensive Coordination Chemistry, Pergaman Press, p. 166 (1987)

2. D.R. Williams, Chem. Rev., 72, 203 (1972).

3. T.M. Sieleciki, J. Liu, S.A. Mousa, A.L. Racanelli, E.A. Hausner, R.R Wexler and R.E. Olson, Bioorg. Med. Chem. Lett., 11, 2210 (2001).

4. P. Sienkiewich, K. Bielawski, A. Bielawska and J. Palka, Environ. Toxicol. Pharmacol, 10, 118 (2005).

5. M. Verma, S.N. Pandeya, K.N. Singh and J.P. Stables, Acta Pharma, 54, 49 (2004).

6. A. Shymal and M.R. Maurya, Synth React. Inorg. Met. Org. Chem., 16, 49 (1986).

7. K. Nakamoto, Infrared and Raman Spectra of Inorganic and Coordination Compunds, John Wiley and Sons, New York, edn. 4 (1986). 